\title{
6. Space Observations of Solar System Objects
}

Solar system objects may be studied in space by two general techniques. Everyone is familiar with the exciting aspects of deep space probes: very high spatial resolution; in situ measurements of particles and fields; in situ chemistry studies by mass spectrographs and gas chromatographs; unique phase angle and occultation opportunities. However, the Solar system can also be studied to great advantage by observatories in orbit around the Earth. The broader spectral range available above the terrestrial atmosphere is as important for planetary studies as it is for investigations of more distant astronomical targets. Both techniques will be discussed in this brief report.

The period from July 1, 1981 to June 30, 1984, was somewhat of a 1411 following the extraordinary preceding three years, in which the two Voyager spacecraft had close encounters with Jupiter and Saturn and their rings and satellites. The highlights of those missions include: the measurement of trace gases in the planetary atmospheres and in Titan's atmosphere with concentrations of order parts per billion by means of infrared spectroscopy; the most active volcanic regime in the Solar system on the satellite Io; the rings of Jupiter; the thick atmosphere of the satellite Titan (with a column abundance approximately 10 times thicker than that of the Earth!); and the detailed structure of Saturn's rings. There were so many discoveries in that brief period that they have not yet been completely assimilated into the body of knowledge that is planetary science. The data have occupied many planetary scientists for the years of the current report period, and will do so for many years to come.

The planet which has been explored most actively during the current report period is Venus. This includes the continued activity of the United States' Pioneer Venus orbiter, which first encountered the planet on December 4, 1978. (In fact, this long-lived mission is currently being considered as a unique means to investiate comet Halley near perihelion in February, 1986, when the comet will be most active, but when it will not be visible from the Earth because of the Sun.) No fewer than four Soviet Venera spacecraft encountered Venus during the report period, including both landers and orbiters. Venera 13 and 14 arrived in March, 1982 and Veneras 15 and 16 arrived in 0ctober, 1983. A superb book has recently been published (Hunter et a].., 1983) summarizing Venus studies up to and including Venera 14. To highlight just one feature of this planet, it has been noted that there has been a recent pulse of $\mathrm{SO}_{2}$ in the atmosphere, which has decayed, and which suggests that volcanism is currently active there.

Veneras 15 and 16 are in 24 hour, near-polar elliptical orbits, with periapses near $60^{\circ} \mathrm{N}$. These orbits are intended to permit the mapping of the northern hemisphere by radar with a resolution of $1.2 \mathrm{~km}$. Preliminary results show a variety of interesting geological features, including ridges and domes. The full import of this exciting mission has not been realized as of the time of writing this report.

At this time, there are a number of missions planned for the near future. The United States is well along with the Galileo orbiter/probe mission to Jupiter, and has begun a Venus Radar Mapper mission. The Voyager flight continues to Uranus (January, 1986) and to Neptune (August, 1989). The Soviet Union will send two probes to comet Halley, with a gravitational assist from Venus. Japan and the European Space Agency will also send probes to this most famous comet, in the first truly international armada of space vehicles with a common target. Arrival wil1 occur in March, 1986.

With regard to Earth orbital astronomy, two United States satellites have made major contributions to planetary studies from space during the report period. 
One satellite made broadband infrared measurements and the other did high resolution ultraviolet spectroscopy.

The brief life of the Infrared Astronomy Satellite (IRAS) occurred entirely within the report period. It was cryogen-limited. However, it completely changed the discipline of planetary science by one class of unexpected discoveries and made numerous other contributions.

The major discovery was that many stars near the Sun exhibit infrared emission in excess of that expected for a star of their respective types. The excesses are attributed to dust rings orbiting the stars. More than forty stars exhibit this characteristic, including Vega and Fomalhaut. These were not among any prominently mentioned leading canidates for extrasolar planets. Once again, scientists have been reminded of the importance of real data, despite the presence of very nice theories. It must be emphasized that the infrared emission is from a low density region of matter, not from a planet like Jupiter or the Earth. However, the list of 40 stars provides an excellent starting point for detailed searches at other wavelengths at higher spatial resolution.

IRAS also made observations of small particles orbiting the Sun. It discovered bands of warm zodiacal dust near the inner edge of the main asteroid belt and observed a dust tail on comet Bowell, outside the orbit of Jupiter. It even managed to discover two comets which subsequently approached the Earth more closely than any other comets this century. One was comet IRAS-Araki-Alcock, the first ever named for a spacecraft.

This leads to the other spectral extreme, with the International Ultraviolet Explorer spacecraft, a joint NASA/ESA/United Kingdom venture that has been operational since 1978 and continues even now. Among the discoveries made with the IUE in the report period was the emission of the $S_{2}$ molecule in comet IRAS-ArakiAlcock. This short-lived molecule was observable frimarily because the comet came so close to the Earth. Other studies with this satellite include the Ioassociated plasma torus around Jupiter, aurorae on Jupiter, atmospheric trace gas compositions on the giant planets and on Venus, and the discovery of an aurora on Uranus. Having pushed the ultraviolet frontier for planetary research below $200 \mathrm{~mm}$ for the first time, the IUE remains exceptionally valuable for studying the Solar system.

It is expected that the next report of this type, covering the period through June, 1987, will be dominated by the first results of the Hubble Space Telescope, a spacecraft with a primary mirror of $2.4 \mathrm{~m}$ diameter, diffraction limited optics throughout the ultraviolet and spectrographs with resolving powers up to $2 \times 10^{5}$. The immediate future holds the promise of extraordinary advances in all areas of Ultraviolet astronomy with the HST, and planetary science will participate fully in this adventure.

\section{Reference}

Huniter, D.M., Colin, L., Donahu, T.M. and Moroz, V.I.: Venus, 1983 